\title{
Actitudes de salud bucodental en relación al nivel socoeconómico en individuos adultos ${ }^{1}$
}

\section{Oral health attitudes related to the socioeconomic level in adults}

\author{
Dhó MS*
}

\section{RESUMEN}

Introducción: Las actitudes intervienen de manera decisiva en la aparición y el desarrollo de las enfermedades bucodentales. El presente trabajo se propone analizar las actitudes de salud bucodental y su relación con el nivel socioeconómico (NSE) en individuos adultos de la Ciudad de Corrientes; Argentina.

Metodología: Se realizó un estudio transversal. A través de una encuesta domiciliaria se recolectó información referente a datos sociodemográficos y a actitudes de salud bucodental utilizando la técnica de observación directa mediante entrevistador "cara a cara". Se determinó el tamaño de la muestra estableciéndose un nivel de confianza del 95\% para la generalización de los resultados (381 individuos). Se aplicó un diseño muestral aleatorio simple para la selección de las viviendas a encuestar, que se complementó con un muestreo no probabilístico por cuotas para la selección de los individuos a entrevistar a partir de los datos proporcionados por el censo 2010. Para el análisis de los datos se empleó el programa SPSS 21.0. Las diferencias según el NSE se analizaron con la prueba Kruskall-Wallis, empleándose pruebas U de Mann-Whitney para valorar las diferencias de a pares.

Resultados: Se halló que los individuos de NSE más bajo presentan actitudes de salud bucodental menos favorables, en particular en lo que se refiere a la actitud frente al dolor dental y a los motivos por los cuales se consulta generalmente al odontólogo.

Conclusiones: Los resultados de este trabajo pueden ser considerados como un recurso importante para diseñar estrategias de intervención que contemplen los determinantes socioculturales del proceso saludenfermedad.

Palabras clave: Salud Oral, encuestas de Salud Dental, comportamientos, odontalgia, gingivitis, consulta odontológica, automedicación.

\section{SUMMARY}

Introduction: Attitudes intervene decisively in the onset and development of oral diseases. This paper analyzes the attitudes toward the oral health and its relationship with socioeconomic status (SES) in adults from the city of Corrientes, Argentina.

Methodology: A cross-sectional study was carried out. Information on sociodemographic and oral health attitudes was collected through a household survey using the technique of direct observation by the "face to face" interviewer. The sample size was determined by establishing a confidence level of $95 \%$ for the generalization

$1 \quad$ Este artículo forma parte de la Tesis de Doctorado de la Universidad Nacional del Nordeste en Odontología bajo la dirección de la Dra. Marta Schufer. Investigación realizada con una beca Doctoral TIPO II del Consejo Nacional de Investigaciones Científicas y Técnicas (CONICET) y la Universidad Nacional del Nordeste (UNNE).

* Odontóloga. Doctora de la Universidad Nacional del Nordeste en Odontología. Becaria Posdoctoral Consejo Nacional de Investigaciones Científicas y Técnicas (CONICET) /Universidad Nacional del Nordeste. Facultad de Odontología. Universidad Nacional del Nordeste. Corrientes. Argentina. 
of results (381 individuals). Simple random sampling design was used for the selection of households to be surveyed, which was supplemented with a non-probability sampling procedure for selecting individuals to be interviewed, based on data provided by the 2010 census. We used a SPSS 21.0 program for the analysis of data. Differences according to the socioeconomic level were analyzed with the Kruskal-Wallis test, using $U$ Mann-Whitney tests to assess differences between pairs.

Results: It was found that individuals from lower socioeconomic level have less favorable attitudes of oral health, particularly in regard to the attitude to dental pain and frequent reason to dentist consultation.

Conclusion: The results of this work can be considered as an important resource to design intervention strategies that address the social and cultural determinants of health-disease process..

Key words: Oral health, Dental Health surveys, behaviors, toothache, gingivitis, dental consultation, selfMedication.

Fecha de recepción: 23 de julio de 2014.

Aceptado para publicación: 21 de octubre de 2014.

Dhó MS. Actitudes de salud bucodental en relación al nivel socoeconómico en individuos adultos. Av. Odontoestomatol 2015; 31 (2): 67-76.

\section{INTRODUCCIÓN}

Las actitudes y los hábitos intervienen de manera decisiva en la aparición y el desarrollo de las enfermedades bucodentales. Su modificación es una estrategia fundamental en la prevención y control de los problemas de salud bucal, con el fin de reducir las intervenciones irreversibles a aquellas situaciones estrictamente necesarias, y siempre que se hayan agotado las medidas preventivas (1).

La caries dental y enfermedad periodontal son enfermedades crónicas y de costoso tratamiento que afectan a las sociedades modernas. En gran medida, su prevención y control dependen del estilo de vida de una persona y de su comportamiento (2).

En la ciudad de Corrientes, varios trabajos han demostrado que la caries dental y las patologías gingivales y periodontales son enfermedades prevalentes (3-5).

La información sistemática sobre las actitudes de salud bucal es necesaria para apoyar la planificación y evaluación de los servicios de salud y para el diseño, elaboración y aplicación de programas de promoción de la salud y prevención de enfermedades bucodentales.
En la estructura de las actitudes, se distinguen tres componentes: a) cognitivo, b) afectivo y c) conductual, este último es el componente activo de la actitud (6).

En el presente trabajo, al hacer referencia a las actitudes de salud bucodental, interesa el componente conductual, específicamente el modo de actuar ante los problemas orales más comunes: dolor dental, sangrado gingival, estrechamente relacionados con los motivos que orientan la búsqueda de atención profesional.

Este estudio se propone describir las actitudes referentes al cuidado de la salud bucal que presentan los individuos adultos que viven en la ciudad de Corrientes y establecer si existen diferencias entre los grupos conformados por distintos niveles socioeconómicos.

\section{MATERIALES Y MÉTODOS}

\section{Población y diseño del estudio}

Se realizó un estudio de tipo transversal en personas de 35 a 44 años de edad que viven en la Ciudad de Corrientes; Argentina. Se focalizó en este grupo de 
edad porque la Organización Mundial de la Salud establece esa franja etaria como el grupo estándar de vigilancia del estado de salud bucodental de los adultos (7). Se incluyeron en el estudio a aquellos individuos que aceptaron voluntariamente contestar las preguntas del formulario de encuesta y se excluyeron a aquellos que no presentaban capacidades físicas y /o mentales para responder el cuestionario y a aquellos que presentaran patologías que impidieran el autocuidado bucodental. La metodología consistió en la aplicación de una encuesta domiciliaria especialmente diseñada para recolectar información referente a las variables de estudio.

\section{Cálculo del tamaño muestral}

A partir de los datos publicados por el Instituto Nacional de Estadísticas y Censos (INDEC) (8), se determinó un universo de 42.242 individuos compuesto por 19.639 hombres y 22.603 mujeres. El tamaño de la muestra se determinó con un nivel de confianza del $95 \%$ y un margen de error de \pm 5 para estimar la proporción en esta población finita. La muestra quedó conformada por 381 individuos, distribuidos en 204 (53,5\%) mujeres y 177 (46,5\%) hombres.

\section{Técnica de muestreo y recolección de datos}

Se aplicó un diseño muestral aleatorio simple para la selección de las viviendas a encuestar, que se complementó con un muestreo no probabilístico por cuotas para la selección de los individuos a entrevistar. Se numeraron todas las manzanas de la ciudad de acuerdo al mapa utilizado en el censo 2.010 proporcionado por la Dirección Provincial de Estadística y Censo y se sortearon con el programa estadístico Epidat 3.1 tantas manzanas como número de individuos se incluyeron en la muestra, de manera tal que se entrevistó a un individuo por manzana para garantizar la mayor cobertura de la varianza de la población. Se seleccionó como vivienda la de numeración más baja en la calle correspondiente al lado norte de la manzana. La selección de los individuos se realizó mediante un muestreo por cuota en función de la variable sexo en el rango de edad seleccionado (35 a 44 años), de acuerdo a la matriz de cuotas que se le proporcionó a cada encuestador.
En cada vivienda seleccionada, se preguntó si había personas con las características establecidas previamente en las cuotas por sexo consignadas en la matriz; en caso de existir dos personas que se ajustasen a las características seleccionadas, se entrevistó al del cumpleaños más próximo; en caso de que no hubiera personas con dichas características, se sustituyó por la vivienda que se encontraba más próxima a la derecha del entrevistador hasta obtener los casos que respondieran a la matriz. Se solicitó a los sujetos su conformidad para participar del estudio, después de explicarles los objetivos del mismo y se garantizó el anonimato y confidencialidad de las respuestas.

Finalizada la encuesta, se entregó a cada participante: un cepillo dental, una pasta dental y un folleto informativo sobre el cuidado de la salud bucal.

\section{Variables de estudio}

- Actitud frente al cuidado de la salud bucodental: Se consideraron tres aspectos de la variable: "actitud frente al dolor dental", "actitud frente al sangrado gingival" y "actitud frente a la consulta odontológica”. Para valorar las actitudes de salud bucodental se redactaron tres preguntas estructuradas de opción múltiple y a cada respuesta se otorgó un valor para su posterior asignación categórica en favorable (2 puntos), regular/aceptable (1 punto), desfavorable (0 punto) (Tabla 1 ).

- Nivel socioeconómico: Para establecer el NSE de los entrevistados se utilizó un índice ya empleado y validado en el ámbito académico (9). En el caso de las amas de casa se adjudicó la categoría ocupacional del jefe de hogar. Los indicadores que se emplean para ubicar a los individuos en cada una de las categorías de educación y de ocupación están especificados en la tabla 2.

El índice de NSE para cada individuo resulta de la combinación de la sumatoria de los puntos obtenidos en los subíndices de nivel educativo y categoría ocupacional. La sumatoria tiene como valor mínimo 2 puntos y, como máximo, 10 puntos. Estos puntos se reúnen luego en las siguientes categorías: bajo; medio-bajo; medio-medio; medio-alto; alto. En el cuadro 1 se establecen los indicadores y las categorías de nivel socioeconómico. 


\section{TABLA 1.- OPERACIONALIZACIÓN DE ACTITUDES DE SALUD BUCODENTAL}

\begin{tabular}{|c|c|c|}
\hline $\begin{array}{l}\text { Actitudes de } \\
\text { salud bucodental }\end{array}$ & $\begin{array}{l}\text { Preguntas para evaluar } \\
\text { las actitudes }\end{array}$ & Respuesta y puntaje otorgado \\
\hline $\begin{array}{l}\text { Actitud frente } \\
\text { al dolor dental }\end{array}$ & $\begin{array}{l}\text { En caso de dolor de diente cqué } \\
\text { es lo primero que Ud. hace? }\end{array}$ & $\begin{array}{l}\text { Se automedica (toma algún medicamento } \\
\text { o remedio/preparado casero) ..................... } \\
\text { Concurre al odontólogo de inmediato ........ } \\
\text { No hace nada, espera que se le pase ......... }\end{array}$ \\
\hline $\begin{array}{l}\text { Actitud frente al } \\
\text { sangrado gingival }\end{array}$ & $\begin{array}{l}\text { Si al cepillarse los dientes o en } \\
\text { cualquier otro momento del día } \\
\text { nota que se le sangran las encías } \\
\text { ¿qué es lo primero que Ud. hace? }\end{array}$ & 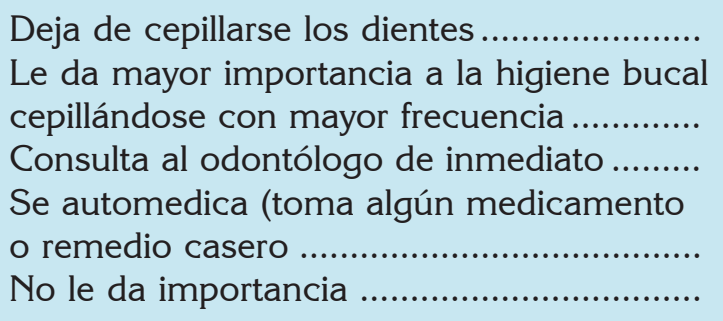 \\
\hline $\begin{array}{l}\text { Actitud frente a la } \\
\text { consulta odontológica }\end{array}$ & $\begin{array}{l}\text { ¿Por qué motivos asiste } \\
\text { generalmente al odontólogo? }\end{array}$ & 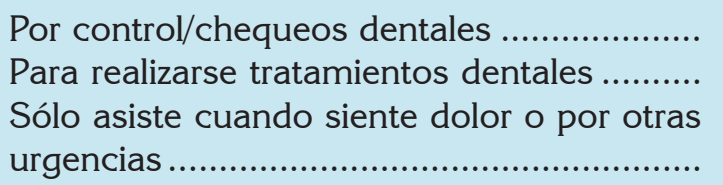 \\
\hline
\end{tabular}

\begin{tabular}{|l|l|l|}
\hline \multicolumn{2}{|c|}{ TABLA 2.- OPERACIONALIZACIÓN DEL NIVEL SOCIOECONÓMICO } \\
\hline Puntos & Nivel educativo & Categoría ocupacional \\
\hline Bajo (1 punto) & No hizo estudios; & Obrero \\
& primaria incompleta & $\begin{array}{l}\text { Desocupado } \\
\text { Beneficiario de planes sociales } \\
\end{array}$ \\
& & $\begin{array}{l}\text { Servicio doméstico } \\
\text { Estudiante }\end{array}$ \\
\hline Medio-bajo (2 puntos) & Primaria completa; & Artesano \\
& secundaria incompleta & Técnico \\
& & Capataz \\
& & Encargado \\
& & Cuenta propia \\
\hline Medio-medio (3 puntos) & Secundaria completa; & Empleado \\
& terciara incompleta & \\
\hline Medio-Alto (4 puntos) & Terciaria completa; & Jefe \\
& universidad incompleta & Profesional independiente \\
\hline Alto (5 puntos) & Universidad completa & Dueño con más de cinco empleados \\
& & Gerente \\
& & Director \\
& & Alto jefe \\
\hline
\end{tabular}




\begin{tabular}{|l|c|}
\hline \multicolumn{2}{|c|}{ CUADRO 1 } \\
\hline Nivel socioeconómico & Puntuación \\
\hline Bajo & De 2 a 3 \\
Medio-bajo & De 4 a 5 \\
Medio-medio & De 6 a 7 \\
Medio-alto & De 8 a 9 \\
Alto & 10 \\
\hline
\end{tabular}

\section{Análisis de los datos}

Se utilizó el programa estadístico SPSS 21.0. Primero se realizó una breve descripción de las características sociodemográficas que presenta la población de estudio, luego se describieron utilizando frecuencias relativas los aspectos referentes a las actitudes de salud bucodental y por último se analizaron las diferencias según el NSE en los tres aspectos de la variable "Actitudes salud bucodental" con pruebas de comparaciones de rangos, específicamente la prueba Kruskall-Wallis, empleándose pruebas U de
Mann-Whitney para valorar las diferencias de a pares. Se empleó un nivel de significación de 0,05.

\section{RESULTADOS}

\section{Caracterización de la población de estudio}

Se entrevistaron 381 individuos con edades comprendidas entre 35 a 44 años, con una edad media de 39,5 años y una desviación estándar de 2,7 años. El sexo se distribuyó de manera proporcional a la población de estudio: 53,5\% (204) mujeres y 46,5\% (177) hombres. Un $66,7 \%$ de la muestra había concluido estudios secundarios. La categoría de ocupación que predomina es "empleado" y el nivel socioeconómico preponderante es el medio-bajo seguido por mediomedio.

Descripción de las actitudes de salud bucodental

En los tres aspectos considerados, predomina una actitud desfavorable (Tabla 3).

\section{TABLA 3.- ACTITUDES DE SALUD BUCODENTAL. EN PORCENTAJES $(\mathrm{N}=381)$}

\section{Actitud frente al dolor dental}

En caso de dolor de diente ¿qué es lo primero que Uv. hace?

- Se automedica (toma algún medicamento o remedio/preparado casero)

- Concurre al odontólogo de inmediato

- No hace nada, espera que se le pase

\section{Actitud frente al sangrado gingival}

Si al cepillarse los dientes o en cualquier otro momento del día nota que le sangran

las encías ¿qué es lo primero que Ud. hace?

- Deja de cepillarse los dientes

- Le da mayor importancia a la higiene bucal, cepillándose con mayor frecuencia

- Consulta al odontólogo de inmediato

- Se automedica (toma algún medicamento o remedio/preparado casero)

- No le da importancia.

\section{Actitud frente a la consulta odontológica}

\section{¿Por qué motivos asiste generalmente al odontólogo?}

- Por control/chequeos dentales

- Para realizarse tratamientos dentales

- Sólo asiste cuando siente dolor o por otras urgencias 


\section{Análisis de las Actitudes de Salud Bucodental en relación al nivel socioeconómico}

Para evaluar las diferencias en las actitudes según el NSE se desarrollaron pruebas de comparación de rangos. Previo a dicho análisis, se elaboraron tablas de contingencia de carácter descriptivo que muestran el porcentaje de respuestas referentes a las actitudes que presentan los individuos según el NSE y se exponen en gráficos. Para el análisis, se agruparon los NSE medio-alto y alto, atento a la escasa población de este último.

\section{Actitud frente al dolor dental}

En los individuos de NSE bajo y medio-bajo, ante una situación de dolor dental predomina una actitud desfavorable (automedicación), mientras que en los individuos de NSE medio-medio y medio-alto/alto predomina una actitud favorable (concurrir al odontólogo de inmediato) (Figura 1).

En tal sentido, al comparar los distintos NSE, se observa que las personas de NSE bajo presentan puntuaciones significativamente menores que las personas de NSE medio-medio ( $U$ de Mann-Withney $=2.760,5, \mathrm{p}<0,000)$ y medio-alto/alto ( $U$ de MannWithney $=1.107,5, \mathrm{p}<0,028$ ), y las personas de NSE medio-bajo muestran puntuaciones significativamente más bajas que las personas de NSE medio-medio ( $U$ de Mann-Withney $=7.549,5, \mathrm{p}<0,002$ ). Las comparaciones restantes no resultaron estadísticamente significativas (Tabla 4).

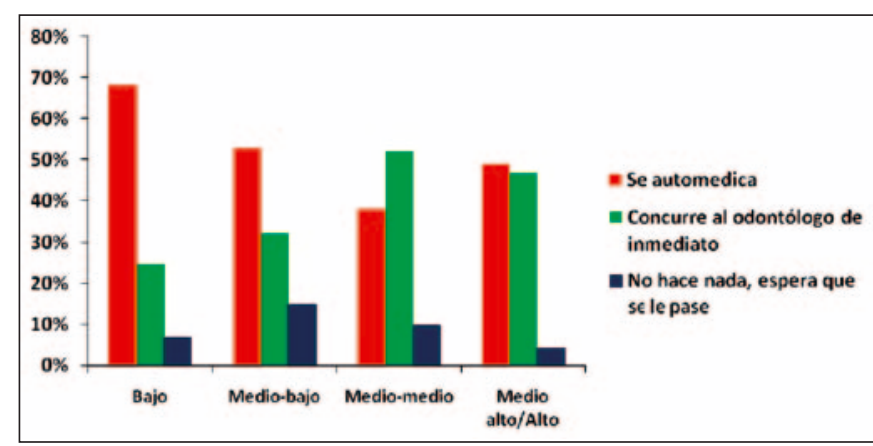

Fig. 1. Actitud frente al dolor dental en relación al NSE.

\section{Actitud frente al sangrado gingival}

En relación a la actitud frente al sangrado gingival, la actitud favorable (consulta odontológica como primera medida), es mayor en los individuos de NSE medio-medio y medio-alto/alto (figura 2); sin embargo, al comparar los distintos NSE no se observan diferencias estadísticamente significativas en las opciones de respuestas seleccionadas por los indivi-

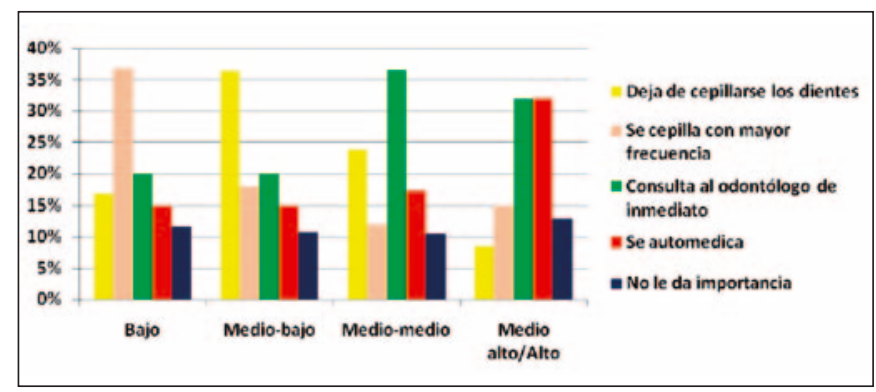

Fig. 2. Actitud frente al sangrado gingival en relación al NSE.

\section{TABLA 4.- RANGOS PROMEDIOS Y PRUEBAS NO PARAMÉTRICAS PARA LA ACTITUD FRENTE AL DOLOR DENTAL SEGÚN EL NSE}

\begin{tabular}{|l|c|c|c|c|c|}
\hline \multicolumn{7}{|c|}{ Nivel socioeconómico } & \multirow{2}{*}{ K-W } \\
\hline & Bajo & Medio-bajo & Medio-medio & Medio-alto/Alto & \\
\hline $\begin{array}{l}\text { En caso de dolor de diente cqué } \\
\text { es lo primero que Ud. hace? }\end{array}$ & $155,26^{\mathrm{a}}$ & $179,84^{\mathrm{a}, \mathrm{b}}$ & $216,07^{\mathrm{c}}$ & $198,40^{\mathrm{b}, \mathrm{c}}$ & \multirow{2}{*}{$18,34^{* * *}$} \\
\hline
\end{tabular}

Nota: Letras distintas indican diferencias estadísticas significativas (prueba $U$ de Mann Withney). $\mathrm{n}=60$, bajo; 140 , medio-bajo; 134 , medio-medio; 47 , medio-alto/alto. $* \mathrm{p}<0,05$; $* * * \mathrm{p}<0,001$. 
duos ante el planteo: "Si al cepillarse los dientes nota que le sangran las encías cqué es lo primero que Ud. hace?" (Tabla 5).

\section{Actitud frente a la consulta odontológica}

En cuanto a la variable motivos por los que asiste al odontólogo, se registró que en los individuos de NSE bajo predomina una actitud desfavorable, pues más del $50 \%$ respondió que solo asiste al odontólogo ante una situación de dolor dental o por otras urgencias odontológicas. La actitud regular/aceptable (asistir al odontólogo para realizarse tratamientos dentales), aumenta proporcionalmente en relación al NSE; no sucediendo lo mismo con la actitud favorable (asistir a la consulta odontológica por control/chequeos dentales), que predomina en los sujetos de NSE medio-bajo (Figura 3).

El examen de los valores de rangos de cada uno de los grupos de NSE indica que los individuos de NSE

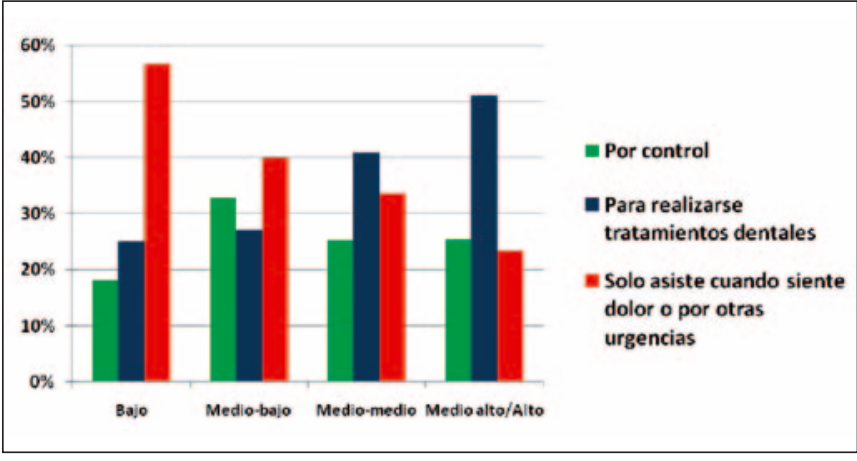

Fig. 3. Actitud frente a la consulta odontológica en relación al NSE.

bajo presentan puntuaciones significativamente menores que las personas de NSE medio-bajo ( $U$ de Mann-Withney $=3.364, \mathrm{p}<0,017)$, medio-medio $(U$ de Mann-Withney =3.139,5, $\mathrm{p}<0,009)$, y medio-alto/ alto $(U$ de Mann-Withney $=983$, $p<0,004)$, sin apreciarse otras diferencias significativas. Las comparaciones restantes no resultaron estadísticamente significativas (Tabla 6).

\section{TABLA 5.- RANGOS PROMEDIOS Y PRUEBAS NO PARAMÉTRICAS PARA LA ACTITUD FRENTE AL SANGRADO GINGIVAL SEGÚN EL NSE}

\begin{tabular}{|l|c|c|c|c|c|}
\hline \multicolumn{7}{|c|}{ Nivel socioeconómico } & \multirow{2}{*}{$K$ - $W$} \\
\hline & Bajo & Medio-bajo & Medio-medio & Medio-alto/Alto & \\
\cline { 1 - 4 } $\begin{array}{l}\text { Si al cepillarse los dietes nota } \\
\text { que le sangran las encías ċqué } \\
\text { es lo primero que Ud. hace? }\end{array}$ & 199,88 & 173,83 & 203,00 & 196,60 & 6.769 \\
\hline
\end{tabular}

Nota: Letras distintas indican diferencias estadísticas significativas (prueba $U$ de Mann Withney). $\mathrm{n}=60$, bajo; 140 , medio-bajo; 134 , medio-medio; 47 , medio-alto/alto. ${ }^{*} \mathrm{p}<0,05 ; * * * \mathrm{p}<0,001$.

\section{TABLA 6.- RANGOS PROMEDIOS Y PRUEBAS NO PARAMÉTRICAS PARA LA ACTITUD FRENTE A LA CONSULTA ODONTOLÓGICA SEGÚN EL NSE}

\begin{tabular}{|l|c|c|c|c|c|}
\hline \multicolumn{7}{|c|}{ Nivel socioeconómico } & \multirow{2}{*}{ K-W } \\
\cline { 1 - 5 } & Bajo & Medio-bajo & Medio-medio & Medio-alto/Alto & \\
\hline $\begin{array}{l}\text { ¿Por qué motivos generalmente } \\
\text { asiste al odontólogo? }\end{array}$ & $155,28^{\mathrm{a}}$ & $195,51^{\mathrm{b}}$ & $195,63^{\mathrm{b}}$ & 209,97 & $9.270^{*}$ \\
\hline
\end{tabular}

Nota: Letras distintas indican diferencias estadísticas significativas (prueba $U$ de Mann Withney). $\mathrm{n}=60$, bajo; 140, medio-bajo; 134 , medio-medio; 47, medio-alto/alto. ${ }^{*} \mathrm{p}<0,05$; $* * * \mathrm{p}<0,001$. 


\section{DISCUSIÓN}

El presente estudio documenta información sobre las actitudes de salud bucodental de los individuos adultos (35-44 años) que viven en la ciudad de Corrientes e ilustra cómo se relacionan estas variables con el nivel socioeconómico.

Se comprobó que el NSE influye en la actitud frente al dolor dental. La prueba de comparación de rangos indica que las personas de NSE bajo presentan una "actitud frente al dolor dental" menos favorable que las personas de NSE medio-medio y medio-alto/ alto. Llama la atención el elevado porcentaje de individuos que ante una situación de dolor dental, como primera medida, se automedicaría. La automedicación es la opción que predomina en los NSE: bajo $(68,3 \%)$ medio-bajo $(52,9 \%)$ y medio alto/alto $(48,9 \%)$, no obstante hay que destacar, que en este último grupo es mínima la diferencia de personas que optan por la automedicación y aquellas que optan por la consulta odontológica $(46,8 \%)$.

Estos resultados coinciden con los registrados en pobladores del Distrito de Independencia de la Provincia de Huaraz, Perú, donde la actitud más prevalente ante el dolor dental es la automedicación, concluyendo que existe una relación inversamente proporcional entre el nivel socioeconómico-cultural y la automedicación en el manejo del dolor dentario (10); y difieren de los obtenidos en un estudio previo realizado en Corrientes donde se reportó que, en caso de dolor dental, el 38,1\% se automedicaría, sin hallarse una asociación importante entre el sexo, la edad y el NSE de los participantes (11). Difieren también los resultados obtenidos por Zhu y col. (2) que reportaron que ante una situación de dolor dental el 30\% de los individuos contestaron que se automedicarían.

La automedicación ante problemas dentales y de otra índole podría verse favorecida por la existencia de numerosos medicamentos de venta libre disponibles no solo en farmacias si no también en otros comercios como ser supermercados y quioscos.

Cabe señalar que no se indagó específicamente por los medicamentos consumidos por los individuos. En tal sentido, son necesarias investigaciones que contemplen este aspecto, así como los efectos se- cundarios de los medicamentos utilizados tanto farmacológicos como tradicionales.

La baja proporción de personas (25\%) de NSE bajo con actitud favorable ante el dolor dental, es decir, que consultan al odontólogo de inmediato, podría atribuirse a problemas relacionados con el acceso a los servicios, la insatisfacción con los servicios prestados en el sector público, los costos que implica la consulta odontológica en el sector privado o problemas personales relacionados con el miedo al dentista.

En relación a la actitud que presentan los encuestados frente al sangrado gingival, un 27,3\% del total indicó que lo primero que haría si le sangran las encías es consultar al odontólogo, un 25,5\% dejarían de cepillarse los dientes, un $18,4 \%$ se cepillaría con mayor frecuencia, un $17,8 \%$ se automedicaría y un $11 \%$ no le daría importancia. Estos resultados difieren de los obtenidos por Zhu y col. (2) que hallaron que el $33 \%$ de los encuestados de 35 a 44 años de edad que viven en zonas urbanas contestaron que se cepillarían los dientes con más frecuencia, el $27 \%$ ignoraría el problema y el $18 \%$ consultaría al odontólogo.

En Corrientes, es preocupante que el 25,5\% señale que dejarían de cepillarse los dientes ante una situación de sangrado gingival. Estos resultados difieren de los hallados por Uysal y col. (12) quienes reportaron que más de la mitad de los participantes creen que el cepillado dental no se debe interrumpir cuando las encías sangran.

En relación a los motivos por los cuales se consulta al odontólogo, sólo un $27 \%$ respondió que asiste para realizarse un control/chequeo dental. Estos resultados coinciden con los hallados en una investigación (12) realizada en pacientes que asistieron a una clínica dental, donde a pesar de que todos los participantes del estudio coincidieron en la importancia de la salud bucal y casi todos reconocieron el beneficio de las visitas dentales regulares, la asistencia dental fue basada en los síntomas $(65,2 \%)$ y en particular provocada por el dolor.

El alto porcentaje de consultas odontológicas solamente ante situaciones de urgencia ha sido reportado en múltiples estudios (13-15). 
En Corrientes, la mayor proporción de individuos que asisten al odontólogo generalmente ante situaciones de "dolor dental u otras urgencias odontológicas" se registró en el NSE bajo (56,7\%). La prueba de comparación de rango señaló que los individuos de NSE bajo presenta actitudes menos favorables hacia la consulta odontológica.

En tal sentido, se ha demostrado que las variables que influyen en la decisión de buscar atención dental preventiva difieren con respecto a las que se asocian con la solicitud de servicios curativos. En el primer caso, la educación, el nivel socioeconómico y los estilos de vida parecen más importantes, mientras que, en el segundo, la necesidad percibida, puede aparecer como el factor desencadenante de la solicitud de servicios (16).

La actitud de realizar controles odontológicos de rutina podría verse influenciada por otros factores como, por ejemplo, tener cobertura social odontológica, conductas ligadas a estilos de vida saludable, experiencia anterior de caries, etc.

\section{CONCLUSIONES}

Las personas de NSE más bajo presentan actitudes de salud bucodental menos favorables, en particular en lo que se refiere a la actitud frente al dolor dental (automedicación) y a los motivos por los cuales se consulta generalmente al odontólogo (dolor/urgencias).

Preocupa la marcada tendencia de automedicación frente al dolor dental que presenta casi la mitad de la población de estudio y su incremento en el NSE bajo. En próximas investigaciones sería importante indagar sobre otros factores intervinientes en este comportamiento.

En atención a estos resultados, los ejes de la implementación de programas de promoción de salud y de prevención de las enfermedades bucodentales, deberían focalizarse en:

- Sensibilizar y educar sobre los riesgos y complicaciones de la automedicación.

- Concienciar sobre la importancia de la consulta odontológica ante el sangrado gingival, para po- der diagnosticar el motivo del mismo y aplicar el tratamiento correspondiente.

- Implementar medidas tendientes a aumentar la concurrencia a los servicios odontológicos con fines de prevención, especialmente en los sectores de la población más desfavorecidos.

\section{AGRADECIMIENTOS}

A la Doctora Marta Schufer, por las observaciones al manuscrito del artículo, y al CONICET y la UNNE, por el financiamiento de la Investigación.

\section{BIBLIOGRAFÍA}

1. Henostroza Haro G. Caries Dental Principios y procedimientos para el diagnóstico. Lima: Editorial Medica Ripano; 2007.

2. Zhu L, Petersen PE, Wang HY, Bian JY, Zhang BX. Oral health knowledge, attitudes and behaviour of adults in China. Int Dent J 2005;55 (4):231-41.

3. Silva NB. Informe estadístico de CPOD y ceod de la Provincia de Corrientes año 2011. Ministerio de Educación. Departamento de Odontología Escolar. [Internet]. [Acceso 24-01-13]. Disponible en: http://www.mecc.gob.ar/PDF/ odontologia/INFORME\%20ESTADISTICO \%20DE\%20CPOD\%20Y\%20ceod\%20DE\%20 LA\%20 PROVINCIA\%20DE\%20CORRIENTESCAP.e\%20INT.-\%20AÑO\%202011.pdf.

4. Dhó MS, Vila VG, Palladino AC. Situación de salud bucal de pacientes mayores de 18 años. Cátedra Práctica Clínica Preventiva I, Facultad de Odontología UNNE, Argentina, 2010. Rev Fac Odontol Univ Antioq 2013;24(2):214-31.

5. Buffil CE; López Vallejos MJ; Cardozo ME. Causas más frecuentes que provocan la extracción dentaria en la población de Corrientes. Rev de la Facultad de Odontología UNNE 2009;II(3):20-4.

6. Rodríguez, A. Psicología Social. México: Trillas; 1991. 
7. Organización Mundial del la Salud. Encuestas de Salud Bucodental. Métodos Básicos. $4^{\mathrm{a}}$ ed. Ginebra: OMS; 1997.

8. Instituto Nacional de Estadística y Censo. Cuadro P2-D. Provincia de Corrientes, departamento Capital. Población total por sexo e índice de masculinidad, según edad en años simples y grupos quinquenales de edad. Año 2010. [Internet]. [Acceso: 04 de julio de 12]. Disponible en: http://www.censo 2010.indec. gov.ar/CuadrosDefinitivos/P2-D_18_21.pdf.

9. Foio, S. El perfil socioeconómico de los ingresantes en la UNNE y su relación con la deserción en el primer año, la retención y el rendimiento académico. Corrientes, Argentina: Secretaría General de Planeamiento. Universidad Nacional del Nordeste. [Internet]. 2003 [Acceso 28 de noviembre de 2007]. Disponible en: http:/ /www.unne.edu.ar/Web/estadistica/temainteres/ Texto/Inf_Ingres/inf_ingres.htm.

10. Aguedo Del Castillo A, Chein Villacampa SA. Relación entre la automedicación para el manejo del dolor dentario y el nivel socioeconómico cultural. Odontol. Sanmarquina 2008;11(2):78-82.

11. Dho MS; Palladino AC; Vila VG. MPSP10 - Actitud frente al dolor dental en pacientes adultos. Rev Fac Med UNNE. 2011;XXXI Supl 1:55.

12. Uysal S, Alev F, Yamalik N, Etikan I. Unmet Need for Education by Professionals and Inconsistency in Knowledge and Behavior of Dental Patients. NY State Dent J 2010;76(3):16-21.

13. Varenne B, Petersen PE, Ouattara S. Oral health behaviour of children and adults in urban and rural areas of Burkina Faso, Africa. Int Dent $\mathrm{J}$ 2006;56(2):61-70.

14. Lian CW, Phing TS, Chan CS, Shin BC, Baharuddin LH, Che'Jalil ZB. Oral health knowledge, attitude and practice among secondary school students in Kuching, Sarawak. Arch Orofacial Sciences 2010; 5(1):9-16.

15. Neamatollahi H, Ebrahimi M. Oral health behavior and its determinants in a group of Iranian students. Indian J Dent Res. 2010;21(1): 84-8.

16. Flores NL, López Cámara V. Factores que influyen en la utilización de los servicios odontológicos. Revisión de la literatura. Revista ADM. 2002;LIX(3):100-9.

\section{CORRESPONDENCIA}

María Silvina Dhó

Facultad de Odontología.

Universidad Nacional del Nordeste.

Corrientes. Argentina.

Correo electrónico: silvinadho@gmail.com 